\title{
Variation in Dopamine D2 and Serotonin 5-HT2A Receptor Genes is Associated with Working Memory Processing and Response to Treatment with Antipsychotics
}

\author{
Giuseppe Blasi' ${ }^{1,2}$, Pierluigi Selvaggi', Leonardo Fazio'2, Linda Antonella Antonucci ${ }^{1,3}$, Paolo Taurisano ${ }^{1,2}$, \\ Rita Masellis', Raffaella Romano', Marina Mancini', Fengyu Zhang ${ }^{4}$, Grazia Caforio', Teresa Popolizio², \\ Jose Apud ${ }^{5}$, Daniel R Weinberger ${ }^{4}$ and Alessandro Bertolino*, 1,6 \\ 'Group of Psychiatric Neuroscience, Department of Basic Medical Sciences, Neuroscience and Sense Organs, University of Bari 'Aldo Moro', \\ Bari, Italy; ${ }^{2}$ IRCCS 'Casa Sollievo della Sofferenza', San Giovanni Rotondo, Italy; ${ }^{3}$ Department of Education Science, Psychology, Communication \\ Science, Aldo Moro University, Bari, Italy; ${ }^{4}$ Lieber Institute for Brain Development, Johns Hopkins Medical Campus, Baltimore, MD, USA; \\ ${ }^{5}$ National Institutes of Health, National Institute of Mental Health, Clinical Brain Disorders Branch, Bethesda, MD, USA; ${ }^{\circ}$ RRED, NORD DTA, \\ F. Hoffman-La Roche Ltd., Basel, Switzerland
}

\begin{abstract}
Dopamine D2 and serotonin 5-HT2A receptors contribute to modulate prefrontal cortical physiology and response to treatment with antipsychotics in schizophrenia. Similarly, functional variation in the genes encoding these receptors is also associated with these phenotypes. In particular, the DRD2 rs $1076560 \mathrm{~T}$ allele predicts a lower ratio of expression of D2 short/long isoforms, suboptimal working memory processing, and better response to antipsychotic treatment compared with the G allele. Furthermore, the HTR2A T allele is associated with lower 5-HT2A expression, impaired working memory processing, and poorer response to antipsychotics compared with the $\mathrm{C}$ allele. Here, we investigated in healthy subjects whether these functional polymorphisms have a combined effect on prefrontal cortical physiology and related cognitive behavior linked to schizophrenia as well as on response to treatment with secondgeneration antipsychotics in patients with schizophrenia. In a total sample of 620 healthy subjects, we found that subjects with the rs $1076560 \mathrm{~T}$ and rs6314 T alleles have greater PMRI prefrontal activity during working memory. Similar results were obtained within the attentional domain. Also, the concomitant presence of the rs $1076560 \mathrm{~T} / \mathrm{rs} 63 \mathrm{I} 4 \mathrm{~T}$ alleles also predicted lower behavioral accuracy during working memory. Moreover, we found that rs 1076560 T carrier/rs63 I 4 CC individuals had better responses to antipsychotic treatment in two independent samples of patients with schizophrenia ( $n=63$ and $n=54$, respectively), consistent with the previously reported separate effects of these genotypes. These results indicate that DRD2 and HTR2A genetic variants together modulate physiological prefrontal efficiency during working memory and also modulate the response to antipsychotics. Therefore, these results suggest that further exploration is needed to better understand the clinical consequences of these genotype-phenotype relationships.

Neuropsychopharmacology (20I5) 40, 1600-1608; doi:I0.I038/npp.20I5.5; published online 28 January 2015
\end{abstract}

\section{INTRODUCTION}

Schizophrenia is a heritable brain disorder whose risk is likely increased by multiple genes adding small effect (McGuffin et al, 2001; Ripke et al, 2013). Dorsolateral prefrontal cortex dysfunction (Callicott et al, 2003) and working memory deficits (Egan et al, 2001) in schizophrenia are also heritable and modulated by interacting genetic variations (Bertolino and Blasi, 2009a). Importantly, individual genetic profiles also impact responses to pharmacological treatment (Blasi and Bertolino, 2006).

* Correspondence: Professor A Bertolino, Department of Basic Medical Science, Neuroscience and Sense Organs, University of Bari 'Aldo Moro', Piazza Giulio Cesare, II, Bari 1-70124, Italy, Tel: +39080 5478572, Fax: +39 080 5593204, E-mail: alessandro.bertolino@uniba.it Received 24 July 2014; revised 28 October 2014; accepted 5 November 2014; article preview online 7 January 2015
Dopamine D2 and serotonin 5-HT2A receptors are particularly relevant in this context. D2 receptors modulate cognitive processing (Seamans and Yang, 2004), and their altered signaling appears to confer liability for abnormal prefrontal response and clinical symptoms in schizophrenia (Abi-Dargham et al, 2000; Durstewitz and Seamans, 2008; Seamans and Yang, 2004). Also, they are involved in the action mechanism of antipsychotics. Furthermore, genetic variation within $D R D 2$ is associated with schizophrenia at genome-wide significance levels (Ripke et al, 2014).

The DRD2 gene (11q23) encodes two isoforms with a mechanism of alternative splicing. The D2 long (D2L) isoform is located mainly postsynaptically, whereas the D2 short (D2S) is mainly a presynaptic autoreceptor (Usiello et al, 2000). In previous reports, we have found that the $\mathrm{T}$ allele of a single-nucleotide polymorphism (SNP) positioned in intron $6(\mathrm{rs} 1076560, \mathrm{G}>\mathrm{T})$ and affecting DRD2 splicing is associated with a lower ratio of expression of 
D2S/D2L compared with the G allele. Furthermore, the T allele is associated with altered working memory prefrontal physiology and behavior (Bertolino et al, 2009b; Zhang et al, 2007). This effect may reflect lower stability of prefrontal response networks during working memory processing (Seamans and Yang, 2004), which is driven by greater D2 postsynaptic signaling putatively associated with the $\mathrm{T}$ allele (Usiello et al, 2000; Zhang et al, 2007). Other findings also suggest association between the $\mathrm{T}$ allele and better clinical response after antipsychotic treatment in patients with schizophrenia (Blasi et al, 2011).

5-HT2A receptors have been implicated in prefrontal physiology and in schizophrenia pathophysiology. For example, 5-HT2A signaling improves prefrontal cognition (Harvey, 2003) and contributes to greater spatial tuning of prefrontal pyramidal neurons during working memory in animal models (Williams et al, 2002). Other investigations have revealed abnormal 5-HT2A expression and binding in patients with schizophrenia (Abi-Dargham, 2007; GonzalezMaeso et al, 2008) as well as a relationship between 5-HT2A binding and psychotic symptoms in antipsychotic-naive patients (Gonzalez-Maeso et al, 2008).

The gene coding for 5-HT2A (HTR2A, 13q14-21) has been weakly associated with schizophrenia (Ayalew et al, 2012; Collins et al, 2012; Sanders et al, 2008). Other investigations have linked genetic variation within HTR2A to response to antipsychotics (Arranz et al, 1998; Chen et al, 2009; Masellis et al, 1998; Olajossy-Hilkesberger et al, 2011). In this gene, a non-synonymous SNP located at exon 3 of HTR2A (rs6314, $\mathrm{C}>\mathrm{T}$, histidine $>$ tyrosine) (Ozaki et al, 1996) has been linked with calcium signaling and function of phospholipases C and D (Hazelwood and Sanders-Bush, 2004; Ozaki et al, 1997), human hippocampal volume and activity (Filippini et al, 2006; Schott et al, 2011), episodic memory performance (de Quervain et al, 2003; Schott et al, 2011), response to treatment with clozapine, and diagnosis of schizophrenia (Arranz et al, 1996, 1998;Masellis et al, 1998; Olajossy-Hilkesberger et al, 2011). Furthermore, the rs6314 T compared with the $\mathrm{C}$ allele predicts lower 5-HT2A expression and inefficient cognitive processing in healthy subjects as well as attenuated improvement after antipsychotic treatment in patients with schizophrenia (Blasi et al, 2013a).

The 5-HT2A receptor interacts with dopamine systems (Di Giovanni et al, 2010; Fink and Gothert, 2007; Lieberman et al, 1998). In particular, some studies indicate that antagonism on 5-HT2A receptors indirectly increases DA release in the prefrontal cortex (Fink and Gothert, 2007). On the other hand, other studies report that 5-HT2A agonists increase but 5-HT2A antagonists decrease prefrontal dopamine efflux (Bortolozzi et al, 2005; Pehek et al, 2006). In general, a complex link between 5-HT2A and dopamine tone appears to modulate brain activity (Di Pietro and Seamans, 2007; Lieberman et al, 1998). Consistently, 5HT2A and D2 receptors are both located on prefrontal pyramidal and nonpyramidal neurons (Fink and Gothert, 2007; Jakab and Goldman-Rakic, 1998, 2000; Seamans and Yang, 2004), and their signaling is also transmitted through the common intraneuronal pathways (Beaulieu, 2012; de Bartolomeis et al, 2013). Overall, these findings suggest that the relationship between D2 and 5-HT2A modulates neuronal function through multiple mechanisms, and that their balanced stimulation is crucial.
The aim of this study was to test whether the combined effect of DRD2 rs1076560 and HTR2A rs6314 is relevant for phenotypes linked to schizophrenia. On the basis of previous findings, we hypothesized that these polymorphisms together modulate cognitive processing and response to treatment with antipsychotics. In earlier studies, the GT and CT genotypes of rs1076560 and rs6314, respectively, were associated with suboptimal cognitive processing in healthy subjects. On the other hand, rs 1076560 GG and rs6314 CT were associated with better response to treatment with antipsychotics in patients with schizophrenia. Therefore, we hypothesized a joint effect of rs107650 GT and rs6314 CT genotypes in predicting suboptimal cognitive processing as well as a joint effect of rs1076560 GG and rs6314 CT genotypes in predicting worse response to antipsychotic treatment.

\section{METHODS}

\section{Prefrontal Physiology}

Subjects. Healthy subjects $(n=620)$ from the region of Puglia, Italy were evaluated with the Structured Clinical Interview (First et al, 1996) for the Diagnostic and Statistical Manual of Mental Disorders to exclude any psychiatric disorder. Individuals were included if they had no history of significant drug or alcohol abuse, no active drug use in the past year, no head trauma with loss of consciousness, and no significant medical condition. All healthy subjects underwent one or more fMRI and cognitive testing procedures described below and were genotyped for DRD2 rs1076560 and HTR2A rs6314.

Genotyping. DRD2 rs1076560 was analyzed with allelespecific PCR primers as previously described (Zhang et al, 2007). Determination of HTR2A rs6314 genotype was conducted using the $5^{\prime}$ exonuclease TaqMan assay as previously described (Blasi et al, 2013a). Both genotypes displayed Hardy-Weinberg equilibrium. Given the low number of homozygous subjects for the T allele for each genotype $(N=8$ for $\mathrm{rs6314}$ and $N=7$ for rs1076560, respectively), they were collapsed in one group with heterozygous individuals ( $\mathrm{T}$ carriers) for all subsequent analyses as previously done (Blasi et al, 2013a; Zhang et al, 2007).

$f M R I$

Subjects and cognitive tasks. Three hundred and twenty-two healthy subjects (169 females, mean \pm SD age: $27.9 \pm 7.7$; IQ: $109.2 \pm 12.2$; handedness: $0.77 \pm 0.37$-Edinburgh Inventory (Oldfield, 1971)) underwent fMRI scanning while performing the two-back working memory task (see Supplementary Materials and Methods) (Blasi et al, 2013a). A part of these individuals was investigated in previous studies by our group addressing association of DRD2 rs1076560 $(N=42)$ (Zhang et al, 2007) and of HTR2A rs6314 $(N=169)$ (Blasi et al, 2013a) with fMRI activity during working memory. There were 196 DRD2 GG/HTR2A CC subjects, 43 DRD2 GG/HTR2A T carriers, 70 DRD2 T carriers/HTR2A CC, and 13 DRD2 $\mathrm{T}$ carriers/HTR2A $\mathrm{T}$ carriers in this sample.

To extend working memory findings to another cognitive domain, 265 healthy subjects (144 females, mean \pm SD age: $25.9 \pm 5.1$; IQ: $110.0 \pm 11.7$; handedness: $0.7 \pm 0.4$ ) were 
scanned while performing the variable attentional control (VAC) task (Blasi et al, 2005). There were 178 DRD2 GG/ HTR2A CC subjects, 33 DRD2 GG/HTR2A T carriers, 43 DRD2 T carriers/HTR2A CC, and 11 DRD2 T carriers/ HTR2A T carriers in this sample. The VAC is an eventrelated design task that has been used in several previous investigations (Blasi et al, 2007, 2010, 2013a; Rasetti et al, 2010) to elicit brain activity during three levels of attentional control (low, intermediate, high) (see Supplementary Materials and Methods).

\section{Data Acquisition and Analysis}

The fMRI data were acquired with a 3.0 Tesla scanner (GE Healthcare) (see Supplementary Materials and Methods) and analyzed using Statistical Parametric Mapping 8 (SPM8) (www.fil.ion.ucl.ac.uk/spm) (see Supplementary Materials and Methods). Pre-determined condition effects at each voxel were calculated using a t-statistic for both the two-back and the VAC tasks. This procedure produced a statistical image for the contrast of two-back $v s$ zero-back (N-back working memory task) and for BOLD responses relative to brain processing of stimuli for each level of attentional control elicited by the VAC (high level-HIGH, intermediate level-INT, and low level-LOW).

After individual processing, the contrast images were used in second-level random-effects models to determine task-specific regional responses at the group level. With regard to the two-back working memory task, analysis of variance (ANOVA) was performed on the contrast of interest, with DRD2 rs1076560 and HTR2A rs6314 genotypes as predictors. We used a statistical threshold of $p<0.05$ and a minimum cluster size $(k)=20$. Family-wise error correction was performed using BA46 within prefrontal cortex, which was our hypothesized region of interest based on previous findings (Blasi et al, 2013a; Zhang et al, 2007). The WFU_PickAtlas (http://fmri.wfubmc.edu/software/pickatlas) was used to build such volume of interest.

With regard to the VAC task, ANOVA was again performed on the contrasts of interest, with DRD2 rs 1076560 and HTR2A rs6314 genotypes as predictors and load as the repeated measure factor. Given that the aim of the VAC analysis was to replicate working memory findings in another cognitive domain, we used as a volume of interest a $10 \mathrm{~mm}$ sphere centered on the peak of activity of the prefrontal cluster associated with a DRD2 rs1076560 by HTR2A rs6314 interaction in the N-back analysis. Thus, we used a statistical threshold of $p<0.05$, minimum cluster size $(k)=20$, familywise error corrected within this volume of interest.

ANOVA was used as appropriate to investigate genotype effects on behavioral data during fMRI.

\section{Behavioral Performance During Working Memory}

Five hundred and nineteen healthy subjects (260 females, mean \pm SD age: $26.9 \pm 7.6$; IQ: $107.8 \pm 12.2$ ) performed the two-back working memory task outside the scanner. Some of these individuals were investigated in previous studies by our group addressing association of DRD2 rs1076560 $(N=88)$ (Zhang et al, 2007) and of HTR2A rs6314 $(N=221)$ (Blasi et al, 2013a) with working memory behavior. There were 327 DRD2 GG/HTR2A CC, 77 DRD2 GG/HTR2A T carriers, 90 DRD2 T carriers/HTR2A CC, and 25 DRD2 T carriers/HTR2A $\mathrm{T}$ carriers in this sample. This task was identical to that performed in the fMRI setting (see above). Performance data were recorded as the number of correct responses (accuracy) and reaction time. ANOVA was used to investigate genotype effects on behavioral data.

\section{Response to Antipsychotic Treatment}

Subjects and designs. Two cohorts were included in this investigation. The first cohort included 63 Caucasian patients with SCID diagnosis of schizophrenia, and they were all from the region of Puglia, Italy (Table 1). Inclusion criteria were the same used for the healthy subjects enrolled in this study (see above). On the basis of DRD2 rs1076560, there were $49 \mathrm{GG}$ and $14 \mathrm{GT}$ individuals, whereas there were $49 \mathrm{CC}$ and $13 \mathrm{CT}$ subjects based on HTR2A rs6314. As per protocol, all patients had not received any psychotropic

Table I Characteristics of the Patients with Schizophrenia Included in the Italian and American Samples to Investigate the Interaction between DRD2 rs 1076560 and HTR2A rs6314 on Response to Antipsychotic Treatment

\begin{tabular}{|c|c|c|c|c|c|c|c|}
\hline $\mathbf{N}$ & Sex & Age & $\begin{array}{l}\text { Premorbid IQ } \\
\text { (WRAT) }\end{array}$ & $\begin{array}{l}\text { Length of illness } \\
\text { (months) }\end{array}$ & $\begin{array}{l}\text { Mean chlorpromazine } \\
\text { equivalents ( } \mathrm{mg} / \text { day) }\end{array}$ & $\begin{array}{l}\text { PANSS scores } \\
\text { at baseline }\end{array}$ & $\begin{array}{l}\text { Drug-free } \\
\text { period }\end{array}$ \\
\hline \multicolumn{8}{|l|}{ Italian sample } \\
\hline $\begin{array}{l}63 \\
29 \text { drug free } \\
34 \text { drug naive }\end{array}$ & $\begin{array}{l}15 \text { females } \\
48 \text { males }\end{array}$ & $28.3 \pm 7.4$ & $102.0 \pm 7.7$ & $59.0 \pm 72.2$ & $621.4 \pm 217$ & $\begin{array}{c}\text { Total: } 103.7 \pm 22.1 \\
\text { Positive: } 25.3 \pm 6.2 \\
\text { Negative: } 25.8 \pm 11.2 \\
\text { General psychopathology: } \\
52.1 \pm 11.9\end{array}$ & $11.9+19.8$ months \\
\hline $\mathbf{N}$ & Sex & Age & $\begin{array}{c}\text { IQ } \\
\text { (WAIS) }\end{array}$ & $\begin{array}{l}\text { Length of illness } \\
\text { (months) }\end{array}$ & $\begin{array}{l}\text { Mean chlorpromazine } \\
\text { equivalents (mg/day) }\end{array}$ & $\begin{array}{l}\text { PANSS scores at } \\
\text { baseline (drug) }\end{array}$ & $\begin{array}{l}\text { PANSS scores at } \\
\text { baseline (placebo) }\end{array}$ \\
\hline \multicolumn{8}{|c|}{ American sample } \\
\hline 54 & $\begin{array}{l}\text { I } 8 \text { females } \\
36 \text { males }\end{array}$ & $27.6 \pm 6.3$ & $91.9 \pm 14.1$ & $80.2 \pm 70.2$ & $593.4 \pm 293.9$ & $\begin{array}{c}\text { Total: } 62.4 \pm \text { | } 4.4 \\
\text { Positive: } 14.4 \pm 4.4 \\
\text { Negative: } 16.7 \pm 5.4 \\
\text { General psychopathology: } \\
31.2 \pm 7.6\end{array}$ & $\begin{array}{c}\text { Total: } 62.9 \pm 14.8 \\
\text { Positive: } 14.4 \pm 4 \\
\text { Negative: } 16.8 \pm 6 \\
\text { General psychopathology: } \\
31.3 \pm 7.9\end{array}$ \\
\hline
\end{tabular}


medication, including benzodiazepines, antidepressants, or mood stabilizers, for at least 1 week or 1 month if they were receiving depot medication prior to study inclusion. All patients then received olanzapine monotherapy for 8 weeks (mean olanzapine dose: $20.9 \pm 7.3 \mathrm{mg}$ ). Titration was allowed for the first 4 weeks, and the dose was then kept constant until week 8 . Symptoms were assessed at study entry (day 0 ) and at day 56 ( 8 weeks) with the positive and negative syndrome scale (PANSS) by only one trained psychiatrist (GC), who was blind to genotype.

The second cohort consisted of 54 partially treatmentresistant patients with schizophrenia who were admitted to the Clinical Brain Disorders Branch schizophrenia inpatient research unit at the National Institutes of Health Clinical Center, Bethesda, MD, USA. All these patients were selfreported Caucasians of European ancestry and were diagnosed with chronic schizophrenia using DSM-IV criteria. All individuals volunteered to participate in a double-blind, placebo-controlled cross-over study with standard doses of atypical antipsychotics, including olanzapine, quetiapine, risperidone, ziprasidone, and aripiprazole (Apud et al, 2012). The program typically enrolled patients who previously had adequate trials of standard antipsychotic medication interventions, which were only partially effective in decreasing their symptoms (see Supplementary Materials and Methods). There were $42 \mathrm{GG}$ and $12 \mathrm{GT}$ according to DRD2 rs1076560, whereas there were $47 \mathrm{CC}$ and 7 CT for HTR2A rs6314 (Table 1).

All patients were taking atypical antipsychotics before their admission. After the initial evaluation period, patients were maintained on a standard dosage of one atypical antipsychotic in an open-label fashion for several weeks before transitioning to the coded trial medication and being tapered from their other medication over a period of 4-7 days. All other medications were discontinued. Subgroup 1 $(N=32)$ underwent a sequence of 4 weeks of coded placebo followed by 4 weeks of a coded standard atypical antipsychotic. Subgroup $2(N=22)$ underwent the inverse sequence; that is, after a similar open medication and taper period, they received a standard atypical antipsychotic treatment for 4 weeks followed by placebo for 4 weeks. Weekly assessments with the PANSS were conducted independently by one of four trained research nurses. Two weeks before starting the double-blind protocol and up to 4 weeks after completing it, these evaluations were performed twice a week (see Supplementary Materials and Methods for further details).

\section{Data Analysis}

The sample size of the two patient groups was relatively small, and it was impossible to test for main effects and interactions as we did for fMRI and behavioral data. Therefore, we investigated the combined effect of DRD2 rs1076560 and HTR2A rs6314 on response to antipsychotic treatment using groups reflecting available multilocus genotypic combinations. Thus, the Italian sample was composed of 14 DRD2 GG/HTR2A CT, 35 DRD2 GG/HTR2A CC, and 14 DRD2 GT/HTR2A CC individuals. None of the subjects had the DRD2 GT/HTR2A CC combination. Therefore, ANCOVA was performed with the genotypic combination as the predictor, and the difference between PANSS scores at 0 and 56 days of olanzapine treatment was the dependent variable. Mean olanzapine dose and PANSS baseline scores were specified as nuisance variables.

In the American sample there were $5 \mathrm{DRD} 2 \mathrm{GG} / \mathrm{HTR} 2 \mathrm{~A} \mathrm{CT}$, 37 DRD2 GG/HTR2A CC, 2 DRD2 GT/HTR2A CC, and 10 DRD2 GT/HTR2A CC individuals. Here, ANCOVA was performed with the genotypic combination as the predictor and the difference between the PANSS last measure on placebo and on antipsychotics as the dependent variable. Mean chlorpromazine equivalents during the trial, order, number of days on placebo, number of days on antipsychotics, PANSS baseline scores on placebo, and PANSS baseline scores on antipsychotics were used as nuisance variables.

\section{RESULTS}

\section{Prefrontal Activity during Cognition in Healthy Subjects}

Genotype groups were matched according to age, gender, IQ, and handedness (all $p>0.1$ ). Furthermore, there were no significant main effects or interaction between rs 1076560 and rs6314 on two-back behavioral data in the fMRI sample when scanned. Thus, genotype effects on brain responses during working memory processing in this sample reflect how the brain processed working memory and not how individuals scored on the test. Consistent with previous studies (Blasi et al, 2013a; Zhang et al, 2007), ANOVA in SPM indicated that DRD2 rs1076560 T carriers have greater activity in the left BA46 at two-back relative to GG individuals $(x=-48, y=40, z=14 ; K=100 ; Z=3.70)$. Similarly, HTR2A rs6314 T carriers have a greater BA46 BOLD response compared with CC subjects $(x=-50$, $y=46, z=10 ; K=26 ; Z=3.26)$. Notably, there was also an interaction between $\mathrm{rs} 1076560$ and rs6314 $(x=-56, y=36$, $z=14 ; K=45 ; Z=3.29$ ) (Figure 1a). Here, post hoc analysis on parameter estimates extracted from the significant cluster in BA46 revealed that individuals carrying the $\mathrm{T}$ allele for rs 1076560 and the $\mathrm{T}$ allele for rs6314 have greater BOLD responses relative to all other genotype configurations (post hoc, Fisher's test, all $p<0.001$ ) (Figure 1b).

In order to extend fMRI working memory findings to another cognitive domain, we investigated the interaction between DRD2 and HTR2A on prefrontal responses during attentional control. With this aim, we centered a $10-\mathrm{mm}$ ROI on the local maxima in which the rs 1076560 by rs6314 interaction was found at two-back $(x=-56, y=36$, $z=14)$. Again, we found that the interaction between rs1076560 and rs6314 is associated with the BOLD response in this brain region $(x=-52, y=34, z=6 ; K=82 ; Z=3.45)$ (Figure 1c). Post hoc analysis on parameter estimates was completely consistent with those of the working memory data in indicating greater BA46 activity in T carriers for rs1076560 and for rs6314 (all $p<0.05$ ) (Figure 1d).

Results of an exploratory, uncorrected analysis investigating further putative combined effects of rs1076560 and rs6314 in the working memory and attentional control networks are presented in Supplementary Table 1.

\section{Behavior during Working Memory}

Genotype groups were matched according to age, gender, IQ, and handedness (all $p>0.1$ ). ANOVA on accuracy data 

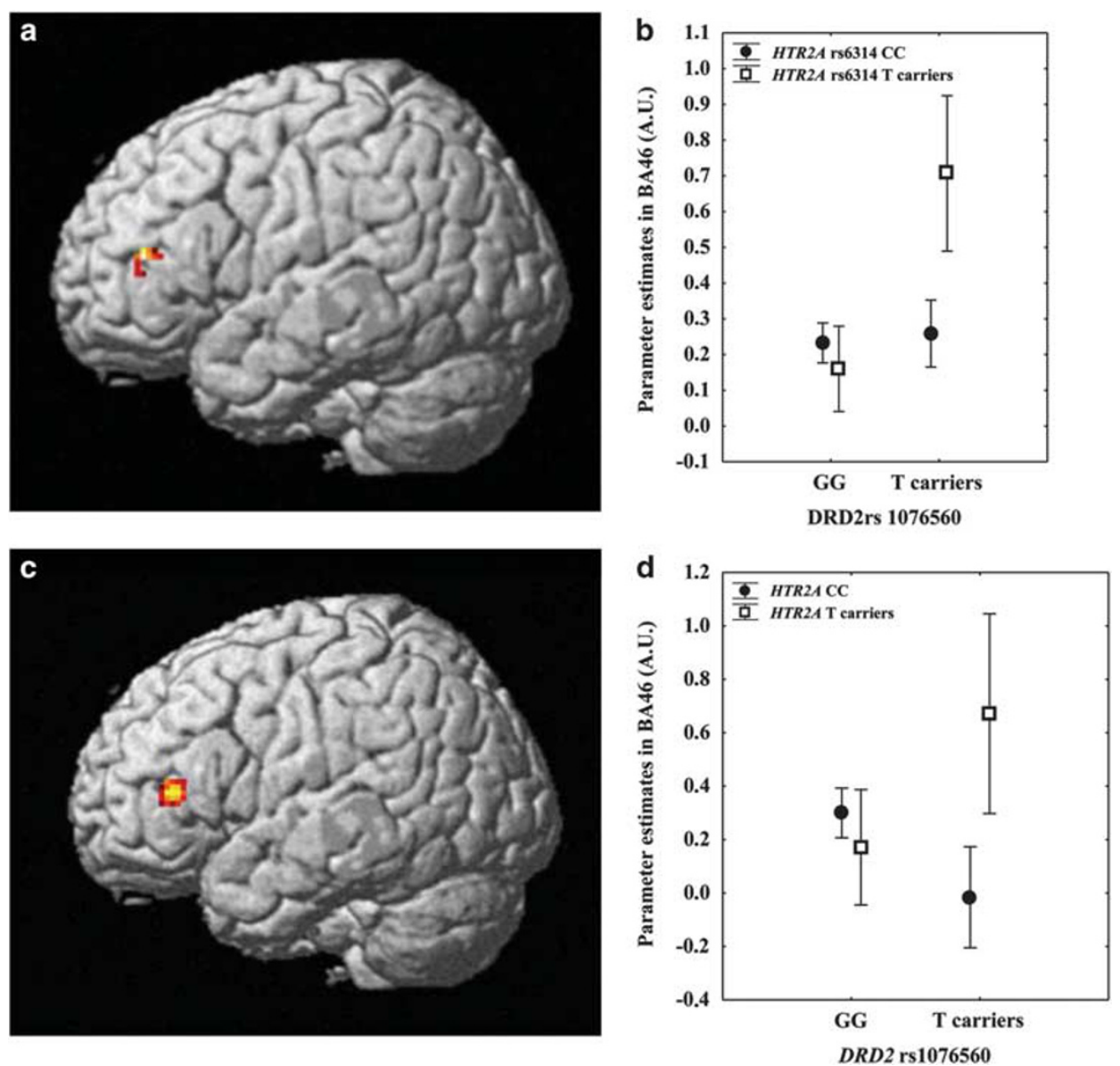

Figure I (a) Render of the brain showing the dorsolateral prefrontal cluster in BA46 associated with the DRD2 rs I076560 by HTR2A rs II30233 interaction during performance of the $\mathrm{N}$-back task, and (b) relative parameter estimates to illustrate the difference in activity between genotype groups. (c) Render of the brain showing the dorsolateral prefrontal cluster in BA46 associated with the DRD2 rs I076560 by HTR2A rs I I30233 interaction during performance of the VAC task, and (d) relative parameter estimates to illustrate the difference in activity between genotype groups.

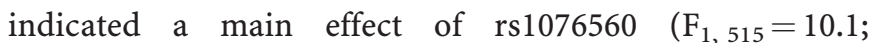
$p=0.001)$ with greater percent correct responses in GG individuals compared with $\mathrm{T}$ carriers. Furthermore, there was a main effect of $\mathrm{rs} 6314\left(\mathrm{~F}_{1,515}=4.8 ; p=0.02\right)$ such that there was greater accuracy in CC compared with T-carrying subjects. Importantly, there was an rs1076560 by rs6314 interaction $\left(\mathrm{F}_{1,515}=10.8 ; p=0.001\right)$. More in detail, subjects carrying the $\mathrm{T}$ allele for rs 1076560 and for rs6314 had a lower percentage of correct responses relative to all other genotype groups (Fisher's post hoc: all $p \leqslant 0.001$ ) (Figure 2).

\section{Response to Treatment with Antipsychotics}

In the Italian sample, groups were matched according to age, gender, premorbid IQ, length of illness, drug-free period, and baseline PANSS scores (all $p>0.15$ ). Mean olanzapine dose during the trial was greater for DRD2 GG/HTR2A CT vs DRD2 GG/HTR2A CC and DRD2 GT/HTR2A CC subjects (all $p<0.04$ ), whereas it did not differ between the latter two groups $(p>0.3)$. ANCOVA with olanzapine mean dose and baseline PANSS scores as nuisance variables indicated a main effect of genotypic combination on the difference in negative symptoms between 0 and 56 days of olanzapine treatment $\left(\mathrm{F}_{2,58}=3,1 ; p=0.05\right)$ (Figure 3a). Fisher's post hoc test revealed that DRD2 GT/HTR2A CC individuals have greater improvement in negative symptoms relative to those with DRD2 GG/HTR2A CC $(p=0.02)$ and DRD2 GG/HTR2A CT $(p=0.005)$ genotypic combinations. There was no difference between DRD2 GG/HTR2A CT and DRD2 GG/ HTR2A CC patients $(p=0.2)$. Furthermore, no main effect of genetic combination was present on total, positive, and general psychopathology PANSS scores (all $p>0.5$ ).

Groups of the American sample did not differ for age, gender, IQ, length of illness, day on placebo and on antipsychotics, and chlorpromazine equivalents during the active phase of the trial. Baseline PANSS scores did not differ as a function of genotypic combination in both the placebo and the antipsychotics arm (all $p>0.2$ ). ANCOVA, with order, PANSS baseline scores, mean chlorpromazine equivalents during the active arm, and days on placebo and days on antipsychotics as nuisance variables indicated a statistical trend for an effect of genotypic combination on positive symptoms scores $\left(\mathrm{F}_{3,44}=2.4 ; p=0.07\right)$ (Figure $3 \mathrm{~b}$ ), which was significant after removing the two individuals with DRD2 GT/HTR2A CT genotypes (Figure 3b) $\left(\mathrm{F}_{2,43}=3.5 ; p=0.03\right)$. Exploratory Fisher's post hoc analysis indicated that DRD2 GT/HTR2A CC subjects had better 


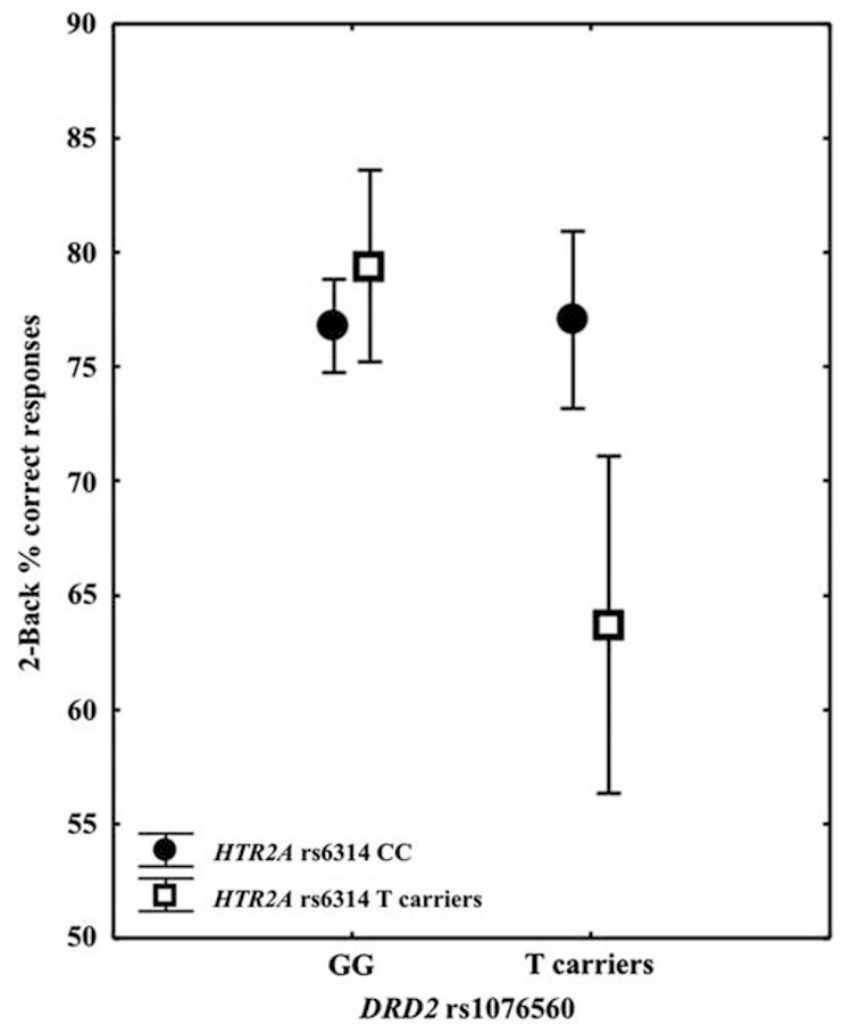

Figure 2 Graph showing the interaction between DRD2 rs 1076560 and HTR2A rs6314 on behavioral accuracy at the two-back working memory task.

responses to antipsychotic treatment compared with $D R D 2$ GG/HTR2A CT individuals $(p<0.007)$. This result is similar to those found in the Italian sample, even if in another symptom domain. No statistically significant difference was present between DRD2 GT/HTR2A CC, DRD2 GT/HTR2A $\mathrm{CT}$, and DRD2 GG/HTR2A CC groups (all $p>0.2$ ). Furthermore, the latter two genotypic configurations had better responses compared with the DRD2 GG/HTR2A CT group (all $p \leqslant 0.04$ ). No statistically significant effects of the genotypic combination was present on PANSS total, negative, and general psychopathology measures (all $p>0.6$ ).

\section{DISCUSSION}

The present results suggest that DRD2 rs1076560 and HTR2A rs6314 together modulate brain activity and behavioral accuracy during working memory in healthy subjects; therefore, these results extend in larger samples previous findings indicating their separate effects. Furthermore, these results also suggest the combined effect of these polymorphisms in the modulation of response to antipsychotic treatment in patients with schizophrenia.

The pooled impact of rs1076560 and rs6314 on working memory processing is manifested in individuals carrying the $\mathrm{T}$ allele in both genes, which is consistent with our earlier results (Blasi et al, 2013a; Zhang et al, 2007). Of note, this combined effect was reproduced when we investigated prefrontal activity associated with attentional processing, a
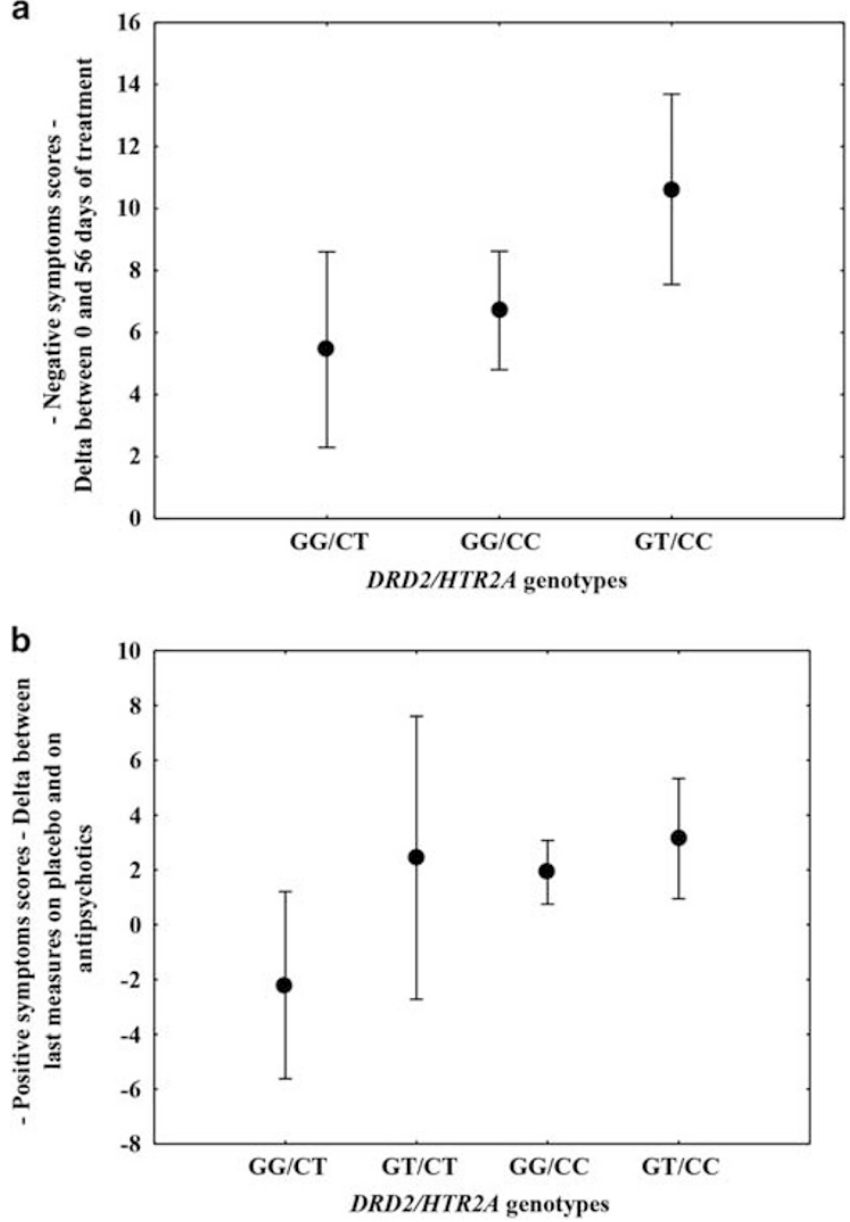

Figure 3 Association of DRD2/HTR2A genotypic combinations scores with response to treatment with antipsychotics. (a) In the Italian sample, there was an association between genotypic combination and response to olanzapine treatment in terms of improvement in negative symptoms. (b) In the American sample, there was a similar association in terms of positive symptoms. See text for statistics.

which is another cognitive domain involved in the pathophysiology of schizophrenia (Blasi et al, 2010; Weickert et al, 2000). Furthermore, we also found that individuals carrying the $\mathrm{T}$ allele in both genes also have lower behavioral accuracy during working memory in a large sample of healthy subjects. Previous studies have indicated that genetic variability in healthy subjects drives variation in prefrontal activity (Blasi et al, 2013b; Huffaker et al, 2009) and lowers behavioral performance (Goldberg et al, 2003). All together, these findings are reminiscent of results in patients with schizophrenia (Callicott et al, 2000), and they have been interpreted as the need for greater recruitment of cortical resources to perform the task with reduced or similar behavioral proficiency (Bertolino and Blasi, 2009a; Manoach, 2003). Consistent with this interpretation, our imaging and behavioral results together suggest that healthy subjects with the $\mathrm{T}$ allele for $D R D 2$ rs1076560 and the $\mathrm{T}$ allele for HTR2A rs6314 have inefficient working memory processing reflected by greater prefrontal activity and lower behavioral accuracy relative to individuals with different genotypic configurations. 
The mechanisms on which these genotype/phenotype relationships rely may be based on molecular effects of DRD2 rs1076560 and HTR2A rs6314 variants on D2 and 5HT2A expression and signaling. The rs1076560 $\mathrm{T}$ allele is associated with relatively greater expression of D2L vs D2S (Zhang et al, 2007) compared with the $G$ allele. This effect may increase D2 postsynaptic signaling (Usiello et al, 2000) and thus decrease the stability of prefrontal response networks and efficiency during working memory processing (Seamans and Yang, 2004). Also, the T allele of HTR2A rs6314 has been linked to less efficient activity and behavior during working memory in a previous report (Blasi et al, 2013a). This effect may be based on association of the $T$ allele with reduced expression of the 5-HT2A receptor (Blasi et al, 2013a), whose signaling appears to facilitate mnemonic processes in prefrontal pyramidal neurons involved in working memory (Williams et al, 2002). Although these findings implicate a separate impact of rs1076560 and rs6314 on prefrontal processing via modulation of D2 and 5-HTA signaling, consistent evidence also indicates that 5HT2A affects dopamine release (Di Giovanni et al, 2010). Based on this knowledge, a possible interpretation of the results of the present study is that the combined genetic effect of DRD2 and HTR2A affects working memory processing via their modulation of D2 and 5-HT2A signaling on activity of prefrontal pyramidal neurons. For example, a genetically mediated 5-HT2A expression on prefrontal dopaminergic terminals may differentially regulate dopamine release (Fink and Gothert, 2007). Furthermore, 5-HT2A may also be differently expressed on prefrontal pyramidal neurons and interneurons as a function of rs6314 genotype. Thus, genetically induced levels of 5-HT2A receptors may in turn interact with lower or greater expression of D2L postsynaptic receptors as a function of $D R D 2$ variation in determining differential levels of stimulation of prefrontal pyramidal neurons or interneurons of relevance for cognitive processing. Another synergistic or alternative mechanism of the combined effect of rs 1076560 and rs6314 on working memory processing is that stimulation of D2 and 5-HT2A receptors implicates intraneuronal adjustments that modulate common pathways including molecules such as AKT1 and GSK3beta (Beaulieu, 2012; de Bartolomeis et al, 2013), whose expression and related genetic variation have been associated with schizophrenia (Blasi et al, 2011, 2013b; Tan et al, 2008). In line with the relevance of common signaling cascades, current evidence also suggests that the cross-talk between D2 and 5-HT2A may be facilitated by the assembly of heteromers (Albizu et al, 2011; de Bartolomeis et al, 2013; Fuxe et al, 2014). Thus, the genetic variation in DRD2 and HTR2A may affect how D2/5HT2A heteromers modulate signaling transduction of relevance for schizophrenia-related phenotypes.

We also find that DRD2 rs1076560 and HTR2A rs6314 together impact the response to antipsychotic treatment in patients with schizophrenia. Specifically, we find that rs1076560 GT/rs6314 CC individuals have better responses to antipsychotics. This association, which is statistically significant in the Italian sample and at a statistical trend level in the American sample, is mainly elicited when comparing rs1076560 GT /rs6314 CC with rs1076560 GG/ rs6314 CT subjects. The rs $1076560 \mathrm{~T}$ allele and the rs6314 C allele separately predicted better response to treatment with second-generation antipsychotics in previous studies (Blasi et al, 2011, 2013a). The fact that genetic variation related to D2 and 5-HT2A signaling have a combined effect on clinical response to second-generation antipsychotics is in line with the antagonistic effects of these drugs on both these receptors. Furthermore, the finding that the therapeutic effect of second-generation antipsychotics is better elicited by specific $D R D 2$ and HTR2A configurations suggests that specific DRD2 and HTR2A alleles may produce patterns of D2 and 5-HT2A expression that are more likely to receive benefit from treatment. The fact that the $\mathrm{T}$ allele of rs 1076560 confers risk for suboptimal prefrontal activity in healthy subjects but that it is also associated with advantage in responding to treatment with antipsychotics requires some explanation. These results are to be interpreted in the context of a possible abnormal dopamine tone and 5-HT2A expression in patients. It is indeed possible that the reduction of dopamine tone and of 5HT2A receptors in the prefrontal cortex of patients with schizophrenia interact with the effects of genetic variation to determine the clinical effects. Regardless of the specific interpretation, these results add that the DRD2/HTR2A interaction is also relevant to modulate clinical response to treatment in patients with schizophrenia.

A limitation of the pharmacogenetic results is the limited size of the two samples used in this study; these samples did not allow us to canonically test for separate main effects of genotypes and their interaction. Larger sample sizes are needed to detect multilocus interaction, or conversely, to demonstrate additivity. Notwithstanding this limitation, we find that the genotype-genotype combination is-or tends to be-associated with response to second-generation antipsychotic treatment in different symptom domains in the two populations investigated, that is, negative symptoms in the Italian population and positive symptoms in the American sample. These results may reflect another limitation of the study. In fact, these independent samples had different characteristics and underwent different protocols of treatment. In particular, the Italian cohort included drugfree (mean drug-free period: $11.9+19.8$ months) or drugnaive, acutely ill patients undergoing an 8-week olanzapinetreatment trial. The American sample was composed of suboptimally treated patients before their admission in a placebo-controlled, cross-over trial. Indeed, the antipsychotic dose levels were significantly tapered in this sample before the standardized psychopharmacological protocol. Thus, it is possible that different trial and population characteristics may reflect on differential detection of genotype effects as clinically elicited. However, the relationships with clinical symptoms that we find in two cohorts suggest that the combined effect of DRD2 and HTR2A polymorphisms is still present even if different methodological approaches are used in limited samples of patients with non-homogeneous characteristics.

In conclusion, the imaging, behavioral, and pharmacogenetic findings of this study implicate the combined effect of DRD2 and HTR2A genetic variations in phenotypes of physiological and clinical interest linked to schizophrenia. They also suggest that the investigation of interactions among multiple genetic characteristics might shed light on the aspects of the physiology of the brain and on the development of brain disorders. 


\section{FUNDING AND DISCLOSURE}

The authors declare no conflict of interest.

\section{ACKNOWLEDGEMENTS}

Professor Bertolino is a full-time employee of Hoffman-La Roche.

\section{REFERENCES}

Abi-Dargham A (2007). Alterations of serotonin transmission in schizophrenia. Int Rev Neurobiol 78: 133-164.

Abi-Dargham A, Rodenhiser J, Printz D, Zea-Ponce Y, Gil R, Kegeles LS et al (2000). Increased baseline occupancy of D2 receptors by dopamine in schizophrenia. Proc Natl Acad Sci USA 97: 8104-8109.

Albizu L, Holloway T, Gonzalez-Maeso J, Sealfon SC (2011). Functional crosstalk and heteromerization of serotonin 5-HT2A and dopamine D2 receptors. Neuropharmacology 61: 770-777.

Apud JA, Zhang F, Decot H, Bigos KL, Weinberger DR (2012). Genetic variation in $\mathrm{KCNH} 2$ associated with expression in the brain of a unique hERG isoform modulates treatment response in patients with schizophrenia. Am J Psychiatry 169: 725-734.

Arranz MJ, Collier DA, Munro J, Sham P, Kirov G, Sodhi M et al (1996). Analysis of a structural polymorphism in the 5-HT2A receptor and clinical response to clozapine. Neurosci Lett 217: 177-178.

Arranz MJ, Munro J, Sham P, Kirov G, Murray RM, Collier DA et al (1998). Meta-analysis of studies on genetic variation in 5-HT2A receptors and clozapine response. Schizophr Res 32: 93-99.

Ayalew M, Le-Niculescu H, Levey DF, Jain N, Changala B, Patel SD et al (2012). Convergent functional genomics of schizophrenia: from comprehensive understanding to genetic risk prediction. Mol Psychiatry 17: 887-905.

Beaulieu JM (2012). A role for Akt and glycogen synthase kinase-3 as integrators of dopamine and serotonin neurotransmission in mental health. J Psychiatry Neurosci 37: 7-16.

Bertolino A, Blasi G (2009a). The genetics of schizophrenia. Neuroscience 164: 288-299.

Bertolino A, Fazio L, Caforio G, Blasi G, Rampino A, Romano R et al (2009b). Functional variants of the dopamine receptor D2 gene modulate prefronto-striatal phenotypes in schizophrenia. Brain 132: 417-425.

Blasi G, Bertolino A (2006). Imaging genomics and response to treatment with antipsychotics in schizophrenia. NeuroRx 3: 117-130.

Blasi G, De Virgilio C, Papazacharias A, Taurisano P, Gelao B, Fazio L et al (2013a). Converging evidence for the association of functional genetic variation in the serotonin receptor $2 \mathrm{a}$ gene with prefrontal function and olanzapine treatment. JAMA Psychiatry 70: 921-930.

Blasi G, Goldberg TE, Elvevag B, Rasetti R, Bertolino A, Cohen J et al (2007). Differentiating allocation of resources and conflict detection within attentional control processing. Eur J Neurosci 25: 594-602.

Blasi G, Mattay VS, Bertolino A, Elvevag B, Callicott JH, Das S et al (2005). Effect of catechol-O-methyltransferase val158met genotype on attentional control. J Neurosci 25: 5038-5045.

Blasi G, Napolitano F, Ursini G, Di Giorgio A, Caforio G, Taurisano P et al (2013b). Association of GSK-3beta genetic variation with GSK-3beta expression, prefrontal cortical thickness, prefrontal physiology, and schizophrenia. Am J Psychiatry 170: 868-876.

Blasi G, Napolitano F, Ursini G, Taurisano P, Romano R, Caforio G et al (2011). DRD2/AKT1 interaction on D2 c-AMP independent signaling, attentional processing, and response to olanzapine treatment in schizophrenia. Proc Natl Acad Sci USA 108: $1158-1163$.
Blasi G, Taurisano P, Papazacharias A, Caforio G, Romano R, Lobianco L et al (2010). Nonlinear response of the anterior cingulate and prefrontal cortex in schizophrenia as a function of variable attentional control. Cereb Cortex 20: 837-845.

Bortolozzi A, Diaz-Mataix L, Scorza MC, Celada P, Artigas F (2005). The activation of 5-HT receptors in prefrontal cortex enhances dopaminergic activity. J Neurochem 95: 1597-1607.

Callicott JH, Bertolino A, Mattay VS, Langheim FJ, Duyn J, Coppola R et al (2000). Physiological dysfunction of the dorsolateral prefrontal cortex in schizophrenia revisited. Cereb Cortex 10: 1078-1092.

Callicott JH, Egan MF, Mattay VS, Bertolino A, Bone AD, Verchinksi B et al (2003). Abnormal fMRI response of the dorsolateral prefrontal cortex in cognitively intact siblings of patients with schizophrenia. Am J Psychiatry 160: 709-719.

Chen SF, Shen YC, Chen CH (2009). HTR2A A-1438G/T102C polymorphisms predict negative symptoms performance upon aripiprazole treatment in schizophrenic patients. Psychopharmacology (Berl) 205: 285-292.

Collins AL, Kim Y, Sklar P, O’Donovan MC, Sullivan PF (2012). Hypothesis-driven candidate genes for schizophrenia compared to genome-wide association results. Psychol Med 42: 607-616.

de Bartolomeis A, Buonaguro EF, Iasevoli F (2013). Serotoninglutamate and serotonin-dopamine reciprocal interactions as putative molecular targets for novel antipsychotic treatments: from receptor heterodimers to postsynaptic scaffolding and effector proteins. Psychopharmacology (Berl) 225: 1-19.

de Quervain DJ, Henke K, Aerni A, Coluccia D, Wollmer MA, Hock C et al (2003). A functional genetic variation of the 5-HT2a receptor affects human memory. Nat Neurosci 6: 1141-1142.

Di Giovanni G, Esposito E, Di Matteo V (2010). Role of serotonin in central dopamine dysfunction. CNS Neurosci Ther 16: 179-194.

Di Pietro NC, Seamans JK (2007). Dopamine and serotonin interactions in the prefrontal cortex: insights on antipsychotic drugs and their mechanism of action. Pharmacopsychiatry 40 Suppl 1: S27-S33.

Durstewitz D, Seamans JK (2008). The dual-state theory of prefrontal cortex dopamine function with relevance to catechol-o-methyltransferase genotypes and schizophrenia. Biol Psychiatry 64: 739-749.

Egan MF, Goldberg TE, Gscheidle T, Weirich M, Rawlings R, Hyde TM et al (2001). Relative risk for cognitive impairments in siblings of patients with schizophrenia. Biol Psychiatry 50: 98-107.

Filippini N, Scassellati C, Boccardi M, Pievani M, Testa C, BocchioChiavetto $\mathrm{L}$ et al (2006). Influence of serotonin receptor $2 \mathrm{~A}$ His452Tyr polymorphism on brain temporal structures: a volumetric MR study. Eur J Hum Genet 14: 443-449.

Fink KB, Gothert M (2007). 5-HT receptor regulation of neurotransmitter release. Pharmacol Rev 59: 360-417.

First MB, Gibbon M, Spitzer RL, Williams JBW (1996). Guide for the Structured Clinical Interview for DSM-IV Axis I DisordersResearch Version. Biometrics Research: New York, NY, USA.

Fuxe K, Borroto-Escuela DO, Tarakanov AO, Romero-Fernandez W, Ferraro L, Tanganelli S et al (2014). Dopamine D2 heteroreceptor complexes and their receptor-receptor interactions in ventral striatum: novel targets for antipsychotic drugs. Prog Brain Res 211: 113-139.

Goldberg TE, Egan MF, Gscheidle T, Coppola R, Weickert T, Kolachana BS et al (2003). Executive subprocesses in working memory: relationship to catechol-O-methyltransferase Val158Met genotype and schizophrenia. Arch Gen Psychiatry 60: 889-896.

Gonzalez-Maeso J, Ang RL, Yuen T, Chan P, Weisstaub NV, Lopez-Gimenez JF et al (2008). Identification of a serotonin/ glutamate receptor complex implicated in psychosis. Nature 452: 93-97.

Harvey JA (2003). Role of the serotonin 5-HT(2A) receptor in learning. Learn Mem 10: 355-362. 
Hazelwood LA, Sanders-Bush E (2004). His452Tyr polymorphism in the human 5-HT2A receptor destabilizes the signaling conformation. Mol Pharmacol 66: 1293-1300.

Huffaker SJ, Chen J, Nicodemus KK, Sambataro F, Yang F, Mattay V et al (2009). A primate-specific, brain isoform of KCNH2 affects cortical physiology, cognition, neuronal repolarization and risk of schizophrenia. Nat Med 15: 509-518.

Jakab RL, Goldman-Rakic PS (1998). 5-Hydroxytryptamine2A serotonin receptors in the primate cerebral cortex: possible site of action of hallucinogenic and antipsychotic drugs in pyramidal cell apical dendrites. Proc Natl Acad Sci USA 95: 735-740.

Jakab RL, Goldman-Rakic PS (2000). Segregation of serotonin 5-HT2A and 5-HT3 receptors in inhibitory circuits of the primate cerebral cortex. J Comp Neurol 417: 337-348.

Lieberman JA, Mailman RB, Duncan G, Sikich L, Chakos M, Nichols DE et al (1998). Serotonergic basis of antipsychotic drug effects in schizophrenia. Biol Psychiatry 44: 1099-1117.

Manoach DS (2003). Prefrontal cortex dysfunction during working memory performance in schizophrenia: reconciling discrepant findings. Schizophr Res 60: 285-298.

Masellis M, Basile V, Meltzer HY, Lieberman JA, Sevy S, Macciardi FM et al (1998). Serotonin subtype 2 receptor genes and clinical response to clozapine in schizophrenia patients. Neuropsychopharmacology 19: 123-132.

McGuffin P, Riley B, Plomin R (2001). Genomics and behavior. Toward behavioral genomics. Science 291: 1232-1249.

Olajossy-Hilkesberger L, Godlewska B, Schosser-Haupt A, Olajossy M, Wojcierowski J, Landowski J et al (2011). Polymorphisms of the 5-HT2A receptor gene and clinical response to olanzapine in paranoid schizophrenia. Neuropsychobiology 64: 202-210.

Oldfield RC (1971). The assessment and analysis of handedness: the Edinburgh inventory. Neuropsychologia 9: 97-113.

Ozaki N, Manji H, Lubierman V, Lu SJ, Lappalainen J, Rosenthal NE et al (1997). A naturally occurring amino acid substitution of the human serotonin 5-HT2A receptor influences amplitude and timing of intracellular calcium mobilization. J Neurochem 68: 2186-2193.

Ozaki N, Rosenthal NE, Pesonen U, Lappalainen J, Feldman-Naim S, Schwartz PJ et al (1996). Two naturally occurring amino acid substitutions of the 5-HT2A receptor: similar prevalence in patients with seasonal affective disorder and controls. Biol Psychiatry 40: 1267-1272.

Pehek EA, Nocjar C, Roth BL, Byrd TA, Mabrouk OS (2006). Evidence for the preferential involvement of 5-HT2A serotonin receptors in stress- and drug-induced dopamine release in the rat medial prefrontal cortex. Neuropsychopharmacology 31: 265-277.

Rasetti R, Mattay VS, Stankevich B, Skjei K, Blasi G, Sambataro F et al (2010). Modulatory effects of modafinil on neural circuits regulating emotion and cognition. Neuropsychopharmacology 35: 2101-2109.

Ripke S, Neale BM, Corvin A, Walters JTR, Farh K, Holmans PA et al (2014). Biological insights from 108 schizophreniaassociated genetic loci. Nature 511: 421-427.

Ripke S, O’Dushlaine C, Chambert K, Moran JL, Kahler AK, Akterin S et al (2013). Genome-wide association analysis identifies 13 new risk loci for schizophrenia. Nat Genet 45: 1150-1159.

Sanders AR, Duan J, Levinson DF, Shi J, He D, Hou C et al (2008). No significant association of 14 candidate genes with schizophrenia in a large European ancestry sample: implications for psychiatric genetics. Am J Psychiatry 165: 497-506.

Schott BH, Seidenbecher CI, Richter S, Wustenberg T, DebskaVielhaber G, Schubert $\mathrm{H}$ et al (2011). Genetic variation of the serotonin 2 a receptor affects hippocampal novelty processing in humans. PLoS One 6: e15984.

Seamans JK, Yang CR (2004). The principal features and mechanisms of dopamine modulation in the prefrontal cortex. Prog Neurobiol 74: 1-58.

Tan HY, Nicodemus KK, Chen Q, Li Z, Brooke JK, Honea R et al (2008). Genetic variation in AKT1 is linked to dopamineassociated prefrontal cortical structure and function in humans. J Clin Invest 118: 2200-2208.

Usiello A, Baik JH, Rouge-Pont F, Picetti R, Dierich A, LeMeur M et al (2000). Distinct functions of the two isoforms of dopamine D2 receptors. Nature 408: 199-203.

Weickert TW, Goldberg TE, Gold JM, Bigelow LB, Egan MF, Weinberger DR (2000). Cognitive impairments in patients with schizophrenia displaying preserved and compromised intellect. Arch Gen Psychiatry 57: 907-913.

Williams GV, Rao SG, Goldman-Rakic PS (2002). The physiological role of 5-HT2A receptors in working memory. J Neurosci 22: 2843-2854.

Zhang Y, Bertolino A, Fazio L, Blasi G, Rampino A, Romano R et al (2007). Polymorphisms in human dopamine D2 receptor gene affect gene expression, splicing, and neuronal activity during working memory. Proc Natl Acad Sci USA 104: 20552-20557.

Supplementary Information accompanies the paper on the Neuropsychopharmacology website (http://www.nature.com/npp) 